\title{
Transdermal hormone therapy in postmenopausal women: A review of metabolic effects and drug delivery technologies
}

\author{
Nathan W Kopper \\ Jennifer Gudeman \\ Daniel J Thompson \\ KV Pharmaceutical, St. Louis, MO, USA
}

Correspondence: Nathan W Kopper KV Pharmaceutical, 2503 South Hanley Road, St. Louis, Missouri 63 I44-2555, USA

Tel + I 3|46456600

$\mathrm{Fax}+$ I 3145670704

Email nkopper@kvph.com

\begin{abstract}
Vasomotor symptoms (VMS) associated with menopause can cause significant discomfort and decrease the quality of life for women in the peri-menopausal and post-menopausal stages of life. Hormone therapy (HT) is the mainstay of treatment for menopausal symptoms and is currently the only therapy proven effective for VMS. Numerous HT options are available to treat VMS, including estrogen-only and estrogen-progestogen combination products to meet the needs of both hysterectomized and nonhysterectomized women. In addition to selecting an appropriate estrogen or estrogen-progestogen combination, consideration should be given to the route of administration to best suit the needs of the patient. Delivery systems for hormone therapy include oral tablets, transdermal patches, transdermal topical (nonpatch) products, and intravaginal preparations. Oral is currently the most commonly utilized route of administration in the United States. However, evidence suggests that oral delivery may lead to some undesirable physiologic effects caused by significant gut and hepatic metabolism. Transdermal drug delivery may mitigate some of these effects by avoiding gut and hepatic first-pass metabolism. Advantages of transdermal delivery include the ability to administer unmetabolized estradiol directly to the blood stream, administration of lower doses compared to oral products, and minimal stimulation of hepatic protein production. Several estradiol transdermal delivery technologies are available, including various types of patches, topical gels, and a transdermal spray.
\end{abstract}

Keywords: estradiol, hormone therapy, menopause, transdermal drug delivery, vasomotor symptoms

\section{Introduction}

Menopause is a biological process whereby ovarian function and production of estradiol decrease, ultimately leading to depletion of ovarian follicles and permanent cessation of menses (Blake 2006). The menopausal transition can occur naturally over a period of several years or spontaneously as a result of surgical removal of the ovaries, chemotherapy, or radiation therapy. Spontaneous natural menopause is identified retrospectively after 12 months of amenorrhea, and reflects a near-complete cessation of ovarian hormone secretion (Soules et al 2001).

As estradiol levels decline during the peri-menopausal and post-menopausal periods, many women experience vasomotor symptoms, breast tenderness, insomnia, migraines, and/or premenstrual dysphoria (Deecher 2005). Vasomotor symptoms (VMS) are the most commonly experienced effects of the menopausal transition and include both hot flashes and night sweats. Long-term estrogen depletion can increase the risk of cardiovascular disease and osteoporosis as well as cause vaginal and vulvar atrophy.

\section{Prevalence}

Vasomotor symptoms affect an estimated $60 \%$ to $80 \%$ of post-menopausal women, but may be experienced by up to $90 \%$ of women during the peri-menopausal stage 
(Rapkin 2007). VMS generally subside within two to five years after menopause; however, up to $30 \%$ of women report VMS into their sixties and almost $10 \%$ report VMS into their seventies. Moderate to severe intensity VMS are reported by $40 \%$ to $60 \%$ of women reporting VMS, and up to $20 \%$ report nearly intolerable symptoms (Bachmann 2005).

\section{Treatment options}

For the treatment of mild VMS, lifestyle changes, either alone or in conjunction with nonprescription remedies, are generally recognized as first-line treatment options (NAMS 2007). Lifestyle modifications include maintaining air temperature as cool as possible to help keep the core body temperature cool and behavioral changes such as exercising regularly, maintaining a healthy body weight, avoiding hot drinks and foods that may trigger hot flashes, and using a variety of relaxation techniques.

Systemic hormone therapy (HT) is the current standard of care for treatment of moderate-to-severe VMS (NAMS 2007) and is the only treatment option that has consistently demonstrated significant reductions in VMS severity and frequency (Fugate and Church 2004; Bachmann 2005; Deecher 2005; Nachtigall et al 2006). In addition, prescription HT is the only treatment currently approved by the United States Food and Drug Administration (FDA) for VMS relief (Bachmann 2005). In the US, hormone therapy is available in oral tablets, transdermal patches, a newly approved transdermal spray, transdermal topical gels and intravaginal preparations (Ansbacher 2001). Systemic therapy with oral and transdermal therapy may be used to treat VMS, vaginal atrophy, and prevention of osteoporosis, although approved indications vary among products. Vaginal dosage forms are generally utilized for local treatment of vulvar and vaginal atrophy; however, one vaginal ring is indicated to treat VMS (Speroff and the United States VR Investigator Group 2003). Estrogenic compounds approved for HT in the US include estradiol, conjugated equine estrogens (CEE), conjugated estrogens (CE), ethinyl estradiol (EE), and estropipate (Minkin 2004).

\section{Alternative treatment options}

Nonprescription remedies such as dietary isoflavones, black cohosh, and vitamin E have been suggested as possible alternatives to HT (Fugate and Church 2004). When studied in randomized, placebo-controlled studies, these remedies have not demonstrated a clinically relevant reduction in VMS (Newton et al 2006). However, a recent study found significant reductions in severity and frequency of hot flashes with a soy isoflavone extract compared to placebo in post-menopausal women experiencing five or more hot flashes per day at baseline after 10 months of treatment (Nahas et al 2007). The soy supplement employed in this trial was highly purified and was tested to ensure that the composition and potency were standardized among all participants. The authors speculate that the lack of benefit noted in other trials of soy products may have been affected by the purity, composition, and lack of standardized manufacturing processes of soy isoflavone extracts.

Off-label use of nonhormonal prescription alternatives including selective serotonin receptor inhibitors (SSRIs), gabapentin, clonidine, or venlafaxine may also be considered when lifestyle changes in conjunction with nonprescription therapies do not provide adequate relief or when contraindications to HT are present (Fugate and Church 2004; Bachmann 2005; Deecher 2005; Nachtigall et al 2006).

\section{Combination HT}

Unopposed estrogen therapy (ET) in nonhysterectomized post-menopausal women has been associated with an increased risk of endometrial hyperplasia, which may be a precursor to the development of endometrial cancer. The duration of unopposed ET is linked to the risk of endometrial cancer. Administration of unopposed ET for 1-2 years increases the risk approximately 2.4-fold (NAMS 2003) whereas administration for 10 years increases the risk 8- to 10-fold (Grady et al 1995). Even after discontinuation of ET, the risk of endometrial cancer appears to remain elevated for at least 5 years (NAMS 2003). Adding a continuous or cyclic progestogen to the estrogen regimen provides endometrial protection, significantly reducing the risk of endometrial hyperplasia and cancer (NAMS 2007; Doherty et al 2007). Progestogens are not required or recommended for hysterectomized women (NAMS 2007).

\section{Randomized controlled trials of $\mathbf{H T}$}

The Heart and Estrogen/progestin Replacement Study II (HERS II) was an open-label continuation of the HERS study, designed to evaluate the cardiovascular effects of $0.625 \mathrm{mg}$ CE plus $2.5 \mathrm{mg}$ medroxyprogesterone acetate (MPA) compared to placebo in postmenopausal women with preexisting cardiovascular disease (CVD) (Grady et al 2002). While no differences in cardiovascular events were noted between the two treatment groups over the 4.1 years of follow-up, posthoc analysis of the data found a trend toward more events in the first year of treatment, but fewer in years 3 through 5 (Hulley et al 1998). HERS-II was designed to determine if 
this reduction in cardiovascular events would be sustained over a longer duration of therapy (Grady et al 2002). The planned follow-up in HERS II was an additional 4 years; however, the trial was stopped after 2.7 years (thus representing a total of 6.8 years of enrollment), due to a lack of protective benefit for HT. No differences were noted between HT and placebo relative to cardiovascular events or death due to CVD, but women receiving HT had a significantly higher rate of nonfatal ventricular arrhythmia compared to those receiving placebo $(\mathrm{p}=0.04)$. In addition, there was no trend toward reduced risk of cardiovascular events with longer duration of HT $(\mathrm{p}=0.18)$.

\section{Women's Health Initiative (WHI)}

The release of the first WHI report in July 2002 occurred within two weeks of publication of HERS II. Like HERS/ HERS II, WHI was designed to determine whether HT had a cardioprotective effect; unlike HERS/HERS II, which enrolled women with pre-existing cardiovascular disease, WHI enrolled healthy women (Rossouw et al 2002). Two different HT regimens were studied: $0.625 \mathrm{mg} \mathrm{CEE}+2.5 \mathrm{mg}$ medroxyprogesterone (MPA) per day in nonhysterectomized women; and $0.625 \mathrm{mg}$ CEE in hysterectomized women (both compared to matching placebo).

The estrogen-progestin WHI study was stopped early due to increased risk of breast cancer, which had been identified at baseline as a primary adverse outcome to be monitored (HR 1.26, 95\% CI 1.00-1.59). Media coverage depicting this $26 \%$ increased risk of breast cancer with estrogenprogestin therapy caused confusion among many patients as well as their healthcare providers, and in many cases made the risk of breast cancer with this regimen appear much greater than the data revealed. For this reason, many experts recommend discussing the risk of breast cancer in terms of absolute risk as compared to relative risk. For example, there were 38 cases of breast cancer among estrogen-progestin users per 10,000 patient years (with 30 cases of breast cancer among placebo users per 10,000 patient years).

Unlike previous observational studies (Henderson et al 1988; Humphrey et al 2002; Stampfer et al 1991; Grodstein et al 1996), the WHI found no cardiovascular protective benefit of the estrogen-progestin combination; in fact, the risk of CVD was slightly increased in women receiving combination estrogen-progestin therapy (EPT) (Rossouw et al 2002). Strokes and venous thromboembolisms (VTEs) were also increased among the combination HT users, which was offset by a reduction in colorectal cancer cases and fractures among combination HT users. No difference was seen in overall mortality between hormone therapy and placebo users.

Published two years later in 2004 was the estrogenonly arm of the WHI. Among estrogen users, there was a significantly higher risk of stroke compared to placebo; again, this was offset by a lower rate of hip fractures compared to placebo users. No significant differences were noted in other clinical outcomes, although there was a trend toward reduced cases of breast cancer among the estrogen users relative to placebo.

The type of women enrolled in WHI has generated criticism as being less than ideal to study primarily due to their age (average of 63.3 years at enrollment) and discouragement of enrolling women experiencing hot flashes at baseline (due to apprehension that women in the placebo group would drop out). To determine whether age at which hormone therapy is commenced may impact the cardiovascular effects, a combined analysis of the WHI trials was recently completed (Rossouw et al 2007). Researchers performed subgroup analysis on women who were 1) less than 10 years; 2) 10-19 years; and 3) 20 or more years past the age of menopause at initiation of HT. For women less than 10 years post-menopausal at study entry, the hazard ratio (HR) of CVD was 0.76 (95\% CI, 0.5-1.16). The HR for women 10-19 years post-menopausal at study entry was $1.1(95 \%$ CI, 0.84-1.45) whereas it was $1.28(95 \%$ CI, 1.03-1.58) for women 20 or more years post-menopausal. Trend analysis revealed a decreased risk of CVD if HT was initiated within 10 years of menopause $(p=0.02)$. However, this trend did not reach the predefined level of statistical significance, which was 0.01 . While p-values of less than 0.05 are often accepted as the benchmark for statistical significance, the authors selected the lower level for this analysis to account for the post hoc nature of this analysis. Nonetheless, these findings, coupled with the realization that the average age at which women were enrolled increased the likelihood of subclinical cardiovascular disease, has generated significant interest and increased the level of comfort with initiating hormone therapy in younger, symptomatic women.

The WHI was the largest controlled clinical trial to date evaluating HT in post-menopausal women; however, this study only evaluated one HT regimen for hysterectomized women (CEE $0.625 \mathrm{mg}$ orally) and one for nonhysterectomized women (CEE $0.625 \mathrm{mg}+$ MPA $2.5 \mathrm{mg}$ ). Thus, some researchers caution that the results found in these studies may not apply to different doses of the same products, other estrogen or progestin compounds, or to products administered by the transdermal or vaginal route (Rossouw et al 2002; Anderson et al 2004). 
Nonetheless, the US FDA requires a black box warning on all post-menopausal HT products regarding the potential risks identified by the WHI (Stephenson 2003). In addition, as a result of HERS, HERS II, and the WHI, HT is no longer recommended solely for the prevention of cardiovascular disease in post-menopausal women (ACOG 2004; Nelson et al 2005; NAMS 2007).

Post-WHI recommendations to utilize the lowest dose for the lowest duration possible have generated interest and the introduction of new low-dose and ultra-low-dose HT products (Stephenson 2003; ACOG 2004; NAMS 2007). Increasing attention is also being paid to the route of administration. Oral products, particularly CE alone or in combination with a progestin (such as those studied in WHI), experienced a large decline in volume following the publication of WHI. (Hersh et al 2004). In recent years, emerging evidence is accumulating regarding the differences between the transdermal and oral route of administration for pharmacokinetic parameters, lipid effects, impact on clotting proteins, and various other processes owing to the avoidance of first-pass metabolism.

\section{Oral vs transdermal: Pharmacokinetic differences}

While both oral and transdermal HT have been proven effective for relief of menopausal symptoms, several differences exist between these routes that may influence safety and patient acceptance of the regimen. Oral administration of estrogen is associated with extensive gut and first-pass liver metabolism as well as significant hepatic stimulation (Balfour and Heel 1990). To overcome these metabolism processes, oral estrogens must be administered in relatively high doses to provide blood levels adequate to reduce menopausal symptoms. Extensive metabolism of oral estrogens results in conversion of a large portion of the dose to estrone and its conjugates, which have less estrogenic activity than estradiol. Significant metabolic conversion of oral estrogens to estrone results in a higher ratio of estrone to estradiol in the blood stream, which is opposite of the physiological levels in premenopausal women (Gleason et al 2005). In addition, some metabolites of conjugated estrogens formed during first-pass metabolism appear to have antiestrogenic or unrecognized pharmacologic activity in the human body (Campagnoli et al 1999). Conversely, transdermal dosage forms deliver estradiol directly to the systemic circulation through the skin, bypassing gut and first-pass hepatic metabolism (Balfour and Heel 1990). Avoidance of gut and liver metabolism via transdermal administration helps maintain an estradiol to estrone ratio similar to that found in premenopausal women (Chetkowski et al 1986). However, the clinical relevance of the estradiol-to-estrone ratio is currently unknown.

Significant variations in the metabolism of oral estrogens results in wide fluctuations in estrogen blood levels throughout the day, potentially resulting in inconsistent control of VMS (Scott et al 1991; Minkin 2004). Transdermal administration provides more consistent blood levels by avoiding the peaks and troughs inherent to oral estrogens.

\section{Lipid effects}

Prior to menopause, endogenous estrogen plays a vital role in lipid metabolism and maintenance of healthy lipid levels. During the peri-menopausal and post-menopausal periods, estrogen depravation contributes to increasing levels of low-density lipoprotein (LDL) cholesterol and triglycerides (TGs) and decreasing levels of high-density lipoprotein (HDL) cholesterol (Menon et al 2006). Exogenous estrogen administration in peri-menopausal and post-menopausal women has been shown to improve lipid profiles (Table 1). However, differences exist between the lipid effects of oral and transdermal estrogens.

Oral and transdermal estrogens have both been shown to increase HDL and decrease LDL and total cholesterol (Sacks and Walsh 1994; Hanggi et al 1997; Shulman et al 2003). However, significant hepatic stimulation with oral estrogens increases production of TGs whereas transdermal estrogens have a neutral effect on TG levels. Thus, the route of

Table I Differential effects of oral and transdermal estrogens on lipids and coagulation factors

\begin{tabular}{lll}
\hline & Oral estrogens & $\begin{array}{l}\text { Transdermal } \\
\text { estrogens }\end{array}$ \\
\hline Total cholesterol & $\downarrow$ & $\downarrow$ \\
HDL-C & $\uparrow$ & $\uparrow$ \\
LDL-C & $\downarrow$ & $\downarrow$ \\
TG & $\uparrow$ & Neutral or $\downarrow$ \\
APC activity & $\downarrow$ & Neutral \\
CRP & $\uparrow$ & Neutral \\
AT III & $\downarrow$ & Neutral \\
Fibrinogen & $\downarrow$ & $\downarrow$ \\
Factor VII activity & Neutral & $\downarrow$ \\
von Willebrand factor & $\uparrow$ & Neutral or $\downarrow$ \\
Prothrombin fragments & $\uparrow$ & Neutral or $\downarrow$ \\
I and 2 & & \\
\hline
\end{tabular}

Abbreviations: HDL-C, high-density lipoprotein cholesterol; LDL-C, low-density lipoprotein cholesterol;TGs, triglycerides;APC, activated protein C; CRP, C-reactive protein;AT III, Antithrombin III. 
estrogen administration should be considered in women with hyperlipidemia as elevated TGs are recognized as an independent risk factor for coronary heart disease (CHD) and increased mortality (Fletcher et al 2005). Additionally, the American Association of Clinical Endocrinologists (AACE) recommends considering transdermal hormone therapy instead of oral therapy for women with elevated TGs or hypertension (AACE 2006).

With respect to LDL, the atherogenicity of LDL may also be influenced by the route of estrogen administration (Wakatsuki et al 2002). TG levels affect the size of LDL particles; higher TG levels contribute to the production of smaller, TG-rich LDL particles (McNamara et al 1992) that are more susceptible to oxidation (Wakatsuki et al 2002). Increased circulating levels of oxidized LDL particles are believed to be associated with atherosclerotic plaque instability and an increased risk of cardiovascular events (Ehara et al 2001). Conversely, by avoiding significant hepatic exposure, transdermal estrogens do not cause increased production of TGs. Thus, despite an overall lowering of total cholesterol and LDL with oral estrogens, increasing production of TGs may reduce some of the lipid benefits inherent to endogenous estrogens (Wakatsuki et al 2002).

The quality of cholesterol molecules can be further affected by the route of estrogen administration. Serum amyloid A (SAA) is an acute-phase protein produced by the liver that is associated with atherosclerosis and vascular inflammation (Liang et al 1996). Oral estrogens increase production of SAA, which when incorporated into HDL particles, can negatively affect the antiatherogenic, antioxidant, and antiinflammatory actions of HDL (Kisilevsky and Subrahmanyan 1992). Conversely, transdermal estrogens do not effect SAA production and thus do not appear to mitigate the positive effects of HDL (Abbas et al 2004).

\section{C-reactive protein}

C-reactive protein (CRP) is an acute-phase protein that has been recognized as a marker for vascular inflammation and atherosclerosis (Pasceri et al 2000). High CRP levels are considered an important predictor of the risk for first myocardial infarction and stroke in otherwise healthy people, independent of other CVD risk factors (Ridker et al 2000; Pradhan et al 2002). Due to the utility of CRP in predicting future cardiovascular disease, the Centers for Disease Control (CDC) and the American Heart Association (AHA) recommend CRP testing and lipid screening in healthy people with an intermediate risk of CVD (Smith et al 2004).
Oral estrogens, via hepatic stimulation, increase production of CRP by the liver (Van Baal et al 1999; Skouby et al 2002). In addition, increases in CRP induced by oral estrogens may be accompanied by a decrease in insulinlike growth factor 1 (IGF-1), an antiinflammatory protein that helps to regulate the inflammatory effects of CRP (Weissberger et al 1991). Conversely, transdermal estrogen does not affect CRP production and may even decrease levels to some degree (Sattar et al 1999; Decensi et al 2002; Strandberg et al 2003; Lacut et al 2003; Vongpatanasin et al 2003). As such, transdermal estrogens may be preferred over oral estrogens in women with cardiovascular disease (CVD) or women at high risk for developing CVD due to the neutral CRP effects of transdermal delivery (Strandberg et al 2003).

\section{Activated protein C (APC)}

Activated protein $\mathrm{C}$ (APC) is a potent thrombin inhibitor that has been recognized as a marker for cardiovascular disease, with reduced activity of APC contributing to an increased risk of cardiovascular events (deVisser et al 1999; Rodeghiero and Tosetto 1999). Oral estrogens appear to decrease the thrombin-inhibiting activity of APC and may contribute to development of APC resistance (Oger 2003). Conversely, transdermal HT administration is associated with minimal effects on APC activity and has not been linked to development of APC resistance (Oger et al 2003; Post et al 2003).

\section{Venous thromboembolism (VTE)}

Exogenous estrogen administration has been shown to affect the levels and activity of certain blood coagulation factors. Antithrombin III (AT III) is a potent coagulation inhibitor that may increase the risk of thrombosis if not present in adequate levels (Bonduki et al 1998). Oral estrogens decrease AT III levels whereas transdermal estrogens appear to have no effect on antithrombin III levels. Numerous other studies have found that compared to oral estrogens, transdermal estrogens have minimal negative impact on other coagulation factors, including fibrinogen, factor VII, von Willebrand factor, and prothrombin fragments $1+2$ (Scarabin et al 1997; Oger et al 2003; Schmidt et al 2006; Menon et al 2006).

An increased risk of venous thromboembolism (VTE) with oral hormone therapy has been shown in both observational and randomized trials (Scarabin et al 2003; Gomes and Deitcher 2004; Canonico et al 2007, 2008). While the VTE risk associated with transdermal estrogens has not been as extensively studied, available evidence suggests that this route may have less propensity toward VTE compared to 
oral administration. This is supported by differences in the production and activity of several clotting factors between these two routes of administration as well as results from a case-control study (Canonico et al 2007).

Recently, VTE risk between oral and transdermal HT was compared in the Estrogen and Thromboembolism Risk (ESTHER) study, which enrolled 881 post-menopausal women (Canonico et al 2007). This case-control study found that the odds ratio of VTE in women using oral HT was 4.2 compared to 0.9 for women receiving transdermal HT. Adjustments for confounding factors such as obesity, family history of VTE, history of varicose veins, education, age at onset of menopause, hysterectomy, and cigarette smoking did not significantly effect these results.

In a previous evaluation of the ESTHER study data, Straczek and colleagues (2005) evaluated the VTE risk of oral and transdermal HT in women with a Factor V Leiden or other prothrombotic mutations. While oral HT increased the risk of VTE 25-fold in women with a prothombotic mutation, transdermal HT had no impact on VTE risk above and beyond the risk already associated with the mutation (Straczek et al 2005) (Figure 1). Randomized controlled trials are needed to confirm the long-term safety of transdermal estrogen, particularly in women with prothrombotic mutations or other risk factors for VTE.

\section{Sex hormone-binding globulin}

Sex hormone-binding globulin (SHBG) is partially responsible for controlling the amount of free testosterone in the blood stream, thereby aiding in the regulation of the androgenic effects of testosterone (Minkin 2004). In excess, SHBG reduces free testosterone levels and thus may decrease libido. Liver metabolism and continued hepatic exposure to estrogen and its metabolites following

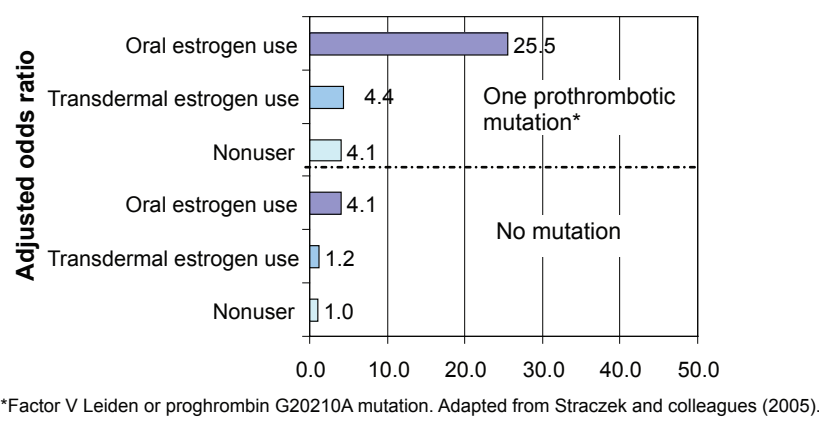

Figure I Comparison of thrombosis risk in women with thrombotic mutations receiving $\mathrm{HT}$.

Abbreviation: HT, hormone therapy. oral estrogen administration increases production of SHBG (Campagnoli et al 1993). Conversely, transdermal estrogen has little effect on SHBG production or circulating free testosterone. Therefore, women with low testosterone or those experiencing sexual dysfunction may benefit from transdermal estrogen rather than oral estrogen (Campagnoli et al 1993; Vehkavaara et al 2000; Samisoe 2001).

\section{Compliance}

Maintaining compliance with HT regimens is an ongoing challenge (Hope and Rees 1995; Kayser et al 2001). One survey of a managed care database found that nearly $75 \%$ of women discontinued HT within 2 years of initiation, no matter the product or dosage form used (Kayser et al 2001). The primary reasons for discontinuation are side effects, including menstrual bleeding, breast tenderness, weight gain, and a fear of cancer (Samsioe 2004). Vaginal bleeding and spotting appear to be lessened by using low-dose or ultra-low-dose products (Mattsson et al 1999; Sturdee et al 2008). By avoiding gut metabolism, gastrointestinal side effects such as nausea, vomiting, and abdominal pain are also less common with transdermal products (Cano 1994; Hirvonen 1997; Archer 2003; Simon 2006). In addition, by allowing use of lower hormone doses, transdermal HT is generally associated with fewer systemic adverse effects, such as vaginal bleeding and breast tenderness (Hivonen 1997).

\section{Transdermal delivery technology}

The clinical benefits of transdermal estradiol delivery are applicable to all transdermal HT products. Several transdermal delivery technologies are utilized for HT products to treat VMS, including patches, gels, an intravaginal ring (Simon and Ravnikar 2007) and a transdermal spray. Transdermal delivery bypasses first-pass hepatic metabolism, thereby allowing administration of lower doses and provides more stable blood levels than oral HT. While equivalent doses of estradiol likely provide equivalent efficacy no matter the delivery system, differences in the application requirements and product formulations may influence patient acceptance of and compliance with these delivery systems.

The first technology used for transdermal delivery of HT was the reservoir patch, which is constructed with a drug reservoir, a backing layer, and an alcohol-containing membrane that controls the rate of drug release into the blood stream (Nachtigall 1995; Musel and Warshaw 2006). Reservoir patches were designed to provide more stable 
estrogen blood levels than oral estrogen, but they have been associated with local irritation rates of up to $46 \%$ (Samsioe 2004). Adhesive composition or the alcohol solvent system is generally thought to be responsible for cutaneous reactions caused by reservoir patches (Musel and Warshaw 2006).

Matrix patches were the next advance in the area of transdermal patch technology. This patch design includes a solution or suspension of active ingredient within a polymer or textile pad that is held in direct contact with the skin. Adhesive is applied only to the perimeter of the patch itself. This design provides a more consistent drug delivery rate than reservoir patches. In addition, matrix patches are generally associated with fewer skin reactions, primarily due to the lack of alcohol and the ability to allow better air circulation to the skin underneath (Simon 2004). The most recent advance in matrix patches was the incorporation of dot matrix technology, which combines the drug and adhesive in a single layer. Dot matrix patches are smaller and thinner than other patches and provide the most consistent drug delivery among transdermal patches with the lowest rates of local irritation among transdermal patches (Reginster et al 2000).

Transdermal patches were designed to improve compliance over oral therapy by providing once- or twice-weekly dosing with a relatively easy to use dosage form. However, some patches, particularly reservoir patches, are associated with relatively high rates of application site reactions including erythema, pruritis, and edema (Samsioe 2004). Additionally, up to $10 \%$ of patches may fall off prematurely, potentially leading to a disruption in the dosing cycle (Erianne and Winter 1997). Patches may also become dirty during the course of wear and leave behind a sticky adhesive residue. Moreover, some women may object to their visual appearance. The release rate of some patches may also be affected by temperature; thus, they should not be applied in a location where they would be exposed to direct sunlight (Estraderm $®$ PI 2005).

The next advance was the development of estradiol topical gels, which were designed to overcome the local irritation caused by transdermal patches (Archer and EstroGel Study Group 2003; Simon et al 2007). In addition to improved tolerability, dose titration is possible with some gel products (Divigel ${ }^{\circledR}$ PI 2007; Simon et al 2007). These products utilize an alcohol base to solvate the skin and allow the estradiol to diffuse through the skin layers. To ensure adequate absorption, gels often require application to the entire arm and necessitate the patient to directly contact the active drug substance, potentially increasing the risk of transfer to another person. In addition, gels must be allowed to dry for several minutes before dressing to prevent transfer of estradiol or the gel itself to clothing. Contact of others with the application site should be avoided for at least 1 hour (Divigel ${ }^{\circledR}$ PI 2007) to 2 hours (Elestrin ${ }^{\circledR}$ PI 2007) to avoid transfer of the active drug. To prevent affecting the absorption of estradiol, the gel application site should be kept dry as long as possible after application; therefore, washing or bathing should occur before application of a gel product.

The latest development in percutaneous drug delivery is an estradiol transdermal spray which recently received FDA approval for treatment of moderate-to-severe VMS (Evamist $^{\mathrm{TM}}$, Ther-Rx Corporation, St. Louis, MO, USA). The transdermal spray technology incorporates estradiol, octisalate, and alcohol into a unique and patented drug delivery system. Upon localized application to the inner forearm, alcohol solvates the stratum corneum and allows the estradiol and octisalate to penetrate the top layer of skin. The alcohol then quickly evaporates, leaving a depot of octisalate and estradiol. Octisalate facilitates the slow descent of estradiol through the epidermis into the dermis, where it is absorbed into the systemic circulation through the capillary beds. Similar to topical gels, this technology provides 24-hour sustained delivery of estradiol while maintaining low blood levels with once-daily dosing (Figure 2). Additionally, application site reactions with estradiol transdermal spray were reported in $1.3 \%$ of patients in a clinical study (Buster et al 2008).

Estradiol transdermal spray provides the advantages of nonoral estradiol delivery while addressing some of the potential drawbacks to topical gels. The spray dries completely and invisibly in a median of 67 seconds and leaves no residue on the skin, allowing women to dress within

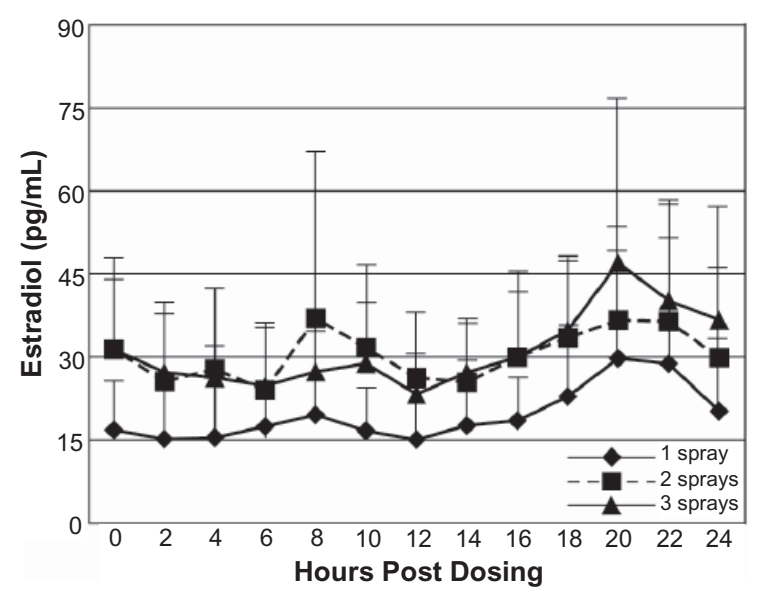

Figure 2. Mean ( $\pm S D$ ) serum estradiol concentrations on day 14 following topical application for 14 days of one, two, or three sprays of Evamist (unadjusted for baseline). Evamist $^{\mathrm{TM}}$ Prescribing Information. Ther-Rx Corporation, St. Louis, Missouri, USA. January 2008. 
2 minutes of application (Buster et al 2008). In addition, washing of the application site is permissible 30 minutes after dosing. The applicator device also eliminates the need for direct contact with the active drug substance, minimizing the risk of transfer to clothing or other people compared to topical gels. To further reduce the risk of transfer to other people, 30 minutes should elapse before allowing contact with the application area.

Estradiol transdermal spray is packaged in a metered-dose pump encased in a plastic housing with a conical bell opening. When pressed against the inner forearm, the applicator device controls the distance, angle, and area of application, thereby reducing the risk of inappropriate application or variability in application technique. Each metered-dose pump is designed to deliver 56 sprays of precisely $90 \mathrm{mcL}$, which contains $1.53 \mathrm{mg}$ of estradiol. As with many transdermal estrogen therapies, administration of higher doses of estradiol to the surface of the skin does not proportionally increase the amount of estradiol absorbed into the systemic circulation. Thus, the amount of estradiol delivered to the systemic circulation is approximately 0.021-, 0.029-, and 0.04-mg per day for the 1-, 2-, and 3-spray doses, respectively (Buster et al 2008). Significant reductions in frequency and severity of moderate-to-severe hot flashes have been demonstrated at all three dosage levels compared to placebo.

The indicated starting dose is 1 spray per day with the option of titrating upward depending upon individual patient response. The ability to titrate the dose allows women to utilize the lowest effective dose, consistent with current recommendations by FDA, American College of Obstetricians and Gynecologists (ACOG) and The North American Menopause Society (NAMS). In addition, as estrogen-related side effects appear to generally be dose-related, administration of lower doses may thus reduce the risk of adverse effects such as breakthrough bleeding and breast tenderness (Lobo 2004).

\section{Conclusion}

Of the various HT options, transdermal delivery is emerging as an increasingly attractive option given the potential for an improved safety profile. While further study is needed, ongoing studies such as the Kronos Early Estrogen Prevention Study (KEEPS) should provide valuable insight regarding the relative safety of transdermal HT compared to oral (Harman et al 2005). Several estradiol transdermal delivery technologies are available for the treatment of moderateto-severe VMS in postmenopausal women. Due to their lack of first-pass hepatic metabolism, transdermal products achieve clinical benefits while minimizing patient exposure to estrogens, which is consistent with the most recent clinical guidelines. The latest development in percutaneous drug delivery, estradiol transdermal spray, offers both the female patient and the clinician a unique option among the hormone therapy armamentarium for the individualized treatment of VMS due to menopause.

\section{Disclosure}

The authors are employed by KV Pharmaceutical, the parent company of Ther-Rx Corporation, which markets Evamist (estradiol transdermal spray).

\section{References}

Abbas A, Fadel PJ, Want Z, et al. 2004. Contrasting effects of oral versus transdermal estrogen on serum amyloid A (SAA) and high-density lipoprotein-SAA in post-menopausal women. Arterioscler Thromb Vasc Biol, 24:e164-67.

ACOG Task Force on Hormone Therapy. 2004. Hormone therapy. Obstet Gynecol, 104(suppl):1s-131s.

[AACE] American Association of Clinical Endocrinologists. 2006. AACE medical guidelines for clinical practice for management of menopause. Endocrinol Pract, 12:315-37.

Anderson GL, Limacher M, Assaf AR, et al; Women's Health Initiative Steering Committee. 2004. Effects of conjugated equine estrogen in post-menopausal women with hysterectomy: the Women's Health Initiative randomized controlled trial. JAMA, 291:1701-12.

Ansbacher R. 2001. The pharmacokinetics and efficacy of different estrogens are not equivalent. Am J Obstet Gynecol,184:255-63.

Archer DF, EstroGel Study Group. 2003. Percutaneous 17-beta-estradiol gel for the treatment of vasomotor symptoms in post-menopausal women. Menopause, 10:516-21.

Archer DF. 2003. Percutaneous 17-beta estradiol gel for the treatment of vasomotor symptoms in post-menopausal women. Menopause, 10:516-21.

Bachmann GA. 2005. Menopausal vasomotor symptoms: a review of causes, effects, and evidence-based treatment options. J Reprod Med, 50:155-65.

Balfour JA, Heel RC. 1990. Transdermal estradiol: a review of its pharmacodynamic and pharmacokinetic properties, and therapeutic efficacy in the treatment of menopausal complaints. Drugs, 40:561-82.

Blake J. 2006. Menopause: evidence-based practice. Best Pract Res Clin Obstet Gynaecol, 20:799-839.

Bonduki CE, Lourenco DM, Baracat E, et al. 1998. Effect of estrogenprogestin hormone replacement therapy on plasma antithrombin III of post-menopausal women. Acta Obstet Gynecol Scand, 77:330-33.

Buster JE, Koltun WD, Pascual MLG, et al. 2008. Low-dose estradiol spray to treat vasomotor symptoms. Obstet Gynecol, 111:1343-51.

Campagnoli C, Ambroggio S, Biglia N, Sismondi P. 1999. Conjugated estrogens and breast cancer risk. Gynecol Endocrinol, 13 (Suppl 6):13-9.

Campagnoli C, Biglia N, Altare F, et al. 1993. Differential effects of oral conjugated estrogens and transdermal estradiol on insulin-like growth factor 1, growth hormone and sex hormone binding globulin serum levels. Gynecol Endocrinol, 7:251-58.

Cano A. 1994. Compliance to hormone replacement therapy in menopausal women controlled in a third level academic centre. Maturitas, 20:91-9.

Canonico M, PluBureau G, Lowe GDO, et al. 2008. Hormone replacement therapy and the risk of venous thromboembolism in postmenopausal women: systematic review and meta-analysis. BMJ, 336:1227-31.

Canonico M, Oger E, Plu-Bureau G, et al. 2007. Hormone therapy and venous thromboembolism among post-menopausal women. Impact of the route of estrogen administration and progestogens: the ESTHER Study. Circulation, 115:840-5. 
Chetkowski RJ, Meldrum DR, Dteingold KA, et al. 1986. Biologic effects of transdermal estradiol. $N$ Engl J Med, 314:1615-20.

Decensi, A, Omodei U, Chris Robertson C, et al. 2002. Effect of transdermal estradiol and oral conjugated estrogens on C-reactive protein in retinoidplacebo trial in healthy women. Circulation, 106:1224-8.

de Visser MCH, Rosendaal FR, Bertina RM. 1999. A reduced sensitivity for activated protein $\mathrm{C}$ in the absence of factor $\mathrm{V}$ Leiden increases the risk of venous thrombosis. Blood, 93:1271-276.

Divigel ${ }^{\circledR}$ PI. 2007. Divigel ${ }^{\circledR}$ prescribing information. Minneapolis, Minnesota, USA: Upsher Smith. June 2007.

Doherty JA, Cushing-Haugen KL, Saltzman BS, et al. 2007. Long-term use of post-menopausal estrogen and progestin hormone therapies and the risk of endometrial cancer. Am J Obstet Gynecol, 197:139e1-e7.

Ehara S, Ueda M, Naruko T, et al. 2001. Elevated levels of oxidized low density lipoprotein show a positive relationship with the severity of acute coronary syndromes. Circulation, 103:1955-160.

Elestrin ${ }^{\circledR}$ PI. 2007. Elestrin ${ }^{\circledR}$ prescribing information. Fairfield, New Jersey, USA: Kenwood Therapeutics. January 2007.

Erianne JA, Winter L, Jr. 1997. Comparison of local tolerability and adhesion of a new matrix system (Menorest ${ }^{\mathbb{}}$ ) for estradiol delivery with an established transdermal membrane system (Estraderm TTS ${ }^{\circledR}$ ). Maturitas, 26:95-101.

Estraderm $^{\circledR}$ PI. 2005. Estraderm ${ }^{\circledR}$ prescribing information. Hanover, NJ, USA: Novartis. January 2005.

Evamist $^{\mathrm{TM}}$ PI. 2008. Evamist ${ }^{\mathrm{TM}}$ prescribing information. St. Louis, MO, USA: Ther-Rx Corporation. January 2008.

Fletcher B, Berra K, Ades P, et al. 2005. AHA Scientific Statement: Managing Abnormal Blood Lipids. A collaborative approach. Circulation, 112:3184-209.

Fugate SE, Church CO. 2004. Nonestrogen treatment modalities for vasomotor symptoms associated with menopause. Ann Pharmacother, 38:1482-99.

Gleason CE, Carlsson CM, Johnson S, et al. 2005. Clinical pharmacology and differential cognitive efficacy of estrogen preparation. Ann NY Acad Sci, 1052:93-115.

Gomes MPV, Deitcher SR. 2004. Risk of venous thromboembolic disease associated with hormonal contraceptives and hormone replacement therapy: a clinical review. Arch Intern Med, 164:1965-76.

Grady D, Gebretsadik T, Kerlikowske K, et al. 1995. Hormone replacement therapy and endometrial cancer risk: a meta-analysis. Obstet Gynecol, 85:304-13.

Grady D, Herrington D, Bittner V, et al. 2002. Cardiovascular disease outcomes during 6.8 years of hormone therapy. Heart and estrogen/progestin replacement study follow-up (HERS II). JAMA, 288:49-57.

Grodstein F, Stampfer MJ, Manson JE, et al. 1996. Post-menopausal estrogen and progestin use and the risk of cardiovascular disease. N Engl J Med, 335:453-61.

Hanggi W, Lippuner K, Riesen W, et al. 1997. Long term influence of different post-menopausal hormone replacement therapy on lipoprotein: A randomized study. Br J Obstet Gynaecol, 104:708-17.

Harman SM, Brinton EA, Cedars M, et al. 2005. KEEPS: The Kronos Early Estrogen Prevention Study. Climacteric, 8:3-12.

Henderson BE, Paganini-Hill A, Ross RK. 1988. Estrogen replacement therapy and protection from acute myocardial infarction. Am J Obstest Gynecol, 159:312-7.

Hersh AL, Stefanick ML, Stafford RS. 2004. National use of postmenopausal hormone therapy. Annual trends and response to recent evidence. JAMA, 291:47-53.

Hing E, Brett KM. 2003. Changes in US prescribing patterns of menopausal hormone therapy, 2001-2003. Obstet Gynecol, 108:33-40.

Hivonen E, Lamberg-Allardt C, Lankinen KS, Geurts P, WilenRosenqvist G. 1997. Transdermal oestradiol gel in the treatment of the climacterium: a comparison with oral therapy. Br J Obstet Gynaecol, 104(Suppl 16):19-25.

Hope S, Rees MCP. 1995. Why do British women start and stop hormone therapy? J Br Menopause Soc, 1:26-7.
Hylley S, Grady D, Bush T, et al. 1998. Randomized trial of estrogen plus progestin for secondary prevention of coronary heart disease in postmenopausal women. JAMA, 280:605-13.

Humphrey LL, Chan BD, Sox HC. 2002. Post-menopausal hormone replacement therapy and the primary prevention of cardiovascular disease. Ann Intern Med, 137:273-84.

Kayser J, Ettinger, B, Pressman A. 2001. Postmenspausal hormone support: discontinuation of raloxifene versus estrogen. Menopause, $8: 328-32$.

Kisilevsky R, Subrahmanyan L. 1992. Serum amyloid A changes high density lipoprotein's cellular affinity: a clue to serum amyloid A's principal function. Lab Invest, 66:778-85.

Lacut K, Oger E, Le Gal G, et al. 2003. Differential effects of oral and transdermal postmenopausal estrogen replacement therapies on C-reactive protein. Thromb Haemost, 90:124-31.

Liang JS, Schreiber BM, Salmona M, et al. 1996. Amino terminal region of acute phase, but not constitutive, serum amyloid A (apoSAA) specifically binds and transports cholesterol into aortic smooth muscle and HepG2 cells. J Lipid Res, 37:2109-16.

Lobo RA. 2004. The rationale for low-dose hormonal therapy. Endocrine, 24:217-21.

Mattsson LA, Bohnet HG, Gredmark T, et al. 1999. Continuous, combined hormone replacement: a randomized comparison of transdermal and oral preparations. Obstet Gynecol, 94:61-5.

McNamara JR, Jenner JL, Li A, et al. 1992. change in LDL particle size is associated with changes in plasma triglyceride concentration. Arterioscler Thromb, 12:1284-90.

Menon DV, Vongpatanasin W. 2006. Effects of transdermal estrogen replacement therapy on cardiovascular risk factors. Treat Endocrinol, 5:37-51.

Minkin MJ. 2002. Considerations in the choice of oral vs transdermal hormone therapy: A review. J Reprod Med, 49:311-20.

Musel AL, Warshaw EM. 2006. Cutaneous reactions to transdermal therapeutic systems. Dermatitis 17:109-22.

Nachtigall LE. 1995. Emerging delivery systems for estrogen replacement: Aspects of transdermal and oral delivery. Am J Obstet Gynecol, 173:993-7.

Nahas EAP, Nahas-Neto J, Orsatti FL, et al. 2007. Efficacy and safety of a soy isoflavone extract in post-menopausal women: A randomized, double-blind, and placebo-controlled study. Maturitas, 58:249-58.

Nelson HD, Humphrey LL, LeBlanc E, et al. 2005. Hormone therapy for the prevention of chronic conditions in post-menopausal women: recommendations from the US Preventive Services Task Force. Ann Intern Med, 142:855-60.

Newton KM, Reed SD, LaCroix AZ, et al. 2006. Treatment of vasomotor symptoms of menopause with black cohosh, multibotanicals, soy, hormone therapy, or placebo. Ann Intern Med, 145:869-79.

Oger E, Alhenc-Gelas M, Lacut K, et al. 2003. Differential effects of oral and transdermal estrogen/progesterone regimens on sensitivity to activated protein $\mathrm{C}$ among post-menopausal women. A randomized trial. Arterioscler Thromb Vasc Biol, 23:1671-76.

Post MS, Christella M, Thomassen LGD, et al. 2003. Effect of oral and transdermal estrogen replacement therapy on hemostatic variables associated with venous thrombosis: A randomized, placebo-controlled study in post-menopausal women. Arterioscler Thromb Vasc Biol, 23:1116-21.

Pradhan AD; Manson JE; RossouwJE, et al. 2002. Inflammatory biomarkers, hormone replacement therapy, and incident of coronary heart disease: prospective analysis from the Women's Health Initiative observational study. JAMA, 288:980-7.

Rapkin AJ. 2007. Vasomotor symptoms in menopause: physiologic condition and central nervous system approaches to treatment. Am J Obstet Gynecol, 196:97-106.

Reginster JY, Donazzolo Y, Brion N, Lins R. 2000. Estradiol pharmacokinetics after transdermal application of patches to post-menopausal women: matrix versus reservoir patches. Climacteric, 3:168-75. 
Ridker PM, Cushman M, Stampfer MJ, et al. 2000. C-reactive protein and other markers of inflammation in the prediction of cardiovascular disease in women. $N$ Engl J Med, 342:836-43.

Rodeghiero F, Tosetto A. 1999. Activated protein C resistance and factor $\mathrm{V}$ Leiden are independent risk factors for venous thromboembolism. Ann Intern Med, 130:643-50.

Rossouw JE, Anderson GL, Prentice RL, et al. 2002. Writing group for the Women's Health Initiative Investigators. Risks and benefits of estrogen plus progestin in healthy post-menopausal women: principal results from the Women's Health Initiative randomized controlled trial. JAMA, 288:321-33.

Rossouw JE, Prentice RL, Manson JE, et al. 2007. Post-menopausal hormone therapy and risk of cardiovascular disease by age and years since menopause. JAMA, 297:1465-77.

Sacks F, Walsh B. 1994. Sex hormones and lipoprotein metabolism. Curr Opin Lipidol, 5:236-40.

Samsioe G. 2004. Transdermal hormone therapy: gels and patches. Climacteric, 7:347-56.

Sattar N, Perera M, Small M, et al. 1999. Hormone replacement therapy and sensitive $\mathrm{C}$-reactive protein concentrations in women with type-2 diabetes. Lancet, 354:487-8.

Scarabin PY, Oger E, Plu-Bureau G, et al. 2003. Differential associatin of oral and transdermal oestrogen-replacement therapy with venous thromboembolism risk. Lancet, 362:428-32.

Schmidt JW, Wollner D, Curcio J, et al. 2006. Hormone replacement therapy in menopausal women: Past problems and future possibilities. Gynecological Endocrinology, 22:564-77.

Scott RT, Jr, Ross B, Anderson C, et al. 1991. Pharmacokinetics of percutaneous estradiol: a crossover study using a gel and a transdermal system in comparison with oral micronized estradiol. Obstet Gynecol, 77:758-64.

Shulman LP, Yankov V, Uhl K. 2003. Safety and efficacy of a continuous once-a-week 17 $\beta$-estradiol/levonorgestrel transdermal system and its effects on vasomotor symptoms and endometrial safety in postmenopausal women: The results of two multicenter, double-blind, randomized, controlled trials. Menopause, 9:195-207.

Simon JA, Bouchard C, Waldebaum A, et al. 2007. Low dose of transdermal estradiol gel for treatment of symptomatic post-menopausal women: a randomized controlled trial. Obstet Gynecol, 109:588-96.

Simon JA, Ravnikar V. 2007. Transdermal estrogen therapy: evidence and update. The Female Patient, November Supplement.

Simon JA. 2004. New options in transdermal administration. OBG Management, November Supplement, 16-9.

Simon JA, Estrasorb Study Group. 2006. Estradiol in micellar nanoparticles: the efficacy and safety of a novel transdermal drug-delivery technology in the management of moderate to severe vasomotor symptoms. Menopause, 13:222-31.

Skouby SO, Gram J, Anderson LF, et al. 2002. Hormone replacement therapy: estrogen and progestin effects on plasma C-reactive protein concentrations. Am J Obstet Gynecol, 186:969-77.

Smith SC, Jr, Anderson JL, Cannon RO 3rd, et al. 2004. CDC/AHA Workshop on markers of inflammation and cardiovascular disease: Application to clinical and public health practice: report from the clinical practice discussion group. Circulation, 110:e550-3.
Soules MR, Sherman S, Parrott E, et al. 2001. Executive summary: stages of reproductive aging workshop (STRAW). Menopause, 8:402-7.

Speroff L, the United States VR Investigator Group. 2003. Efficacy and tolerability of a novel estradiol vaginal ring for relief of menopausal symptoms. Obstet Gynecol, 102:823-34.

Stampfer MJ, Colditz GA, Willett WC, et al. 1991. Post-menopausal estrogen therapy and cardiovascular disease: ten year follow-up from the nurses' health study. N Engl J Med, 325:756-62.

Stampfer MJ, Willett WC, Colditz GA, et al. 1985. A prospective study of post-menopausal estrogen therapy and coronary heart disease. $N$ Engl J Med, 313:1044-9.

Stephenson J. 2003. FDA orders estrogen safety warnings: agency offers guidance for HRT use. JAMA, 289:537-8.

Straczek C, Oger E, Yon de Jonage-canonico MB, et al. 2005. Prothrombotic mutations, hormone therapy and venous thromboembolism among post-menopausal women. Circulation, 112:3495-500.

Strandberg TE, Olavi Ylikorkala O, Tikkanen MJ. 2003. Differing effects of oral and transdermal hormone replacement therapy on cardiovascular risk factors in healthy postmenopausal women. Am J Cardiol, 92:212-4.

Stumpf PG. 1990. Pharmacokinetics of estrogen. Obstet Gyneco, 75(Suppl):9s-17s

Sturdee D, Archer D, Rakov V, et al. 2008. Ultra-low-dose continuous combined estradiol and norethisterone acetate: improved bleeding profile in post-menopausal women. Climacteric, 11:63-73.

Sturdee DW, Archer DF, Rakov V, et al. 2008. Ultra-low-dose continuous combined estradiol and norethisterone acetate: improved bleeding profile in post-menopausal women. Climacteric, 11:68-73.

The North American Menopause Society. 2007. Estrogen and progestogen use in peri- and post-menopausal women: March 2007 position statement of the North American Menopause Society. Menopause, $14: 168-82$.

[NAMS] The North American Menopause Society. 2003. Role of progestogen in hormone therapy for post-menopausal women: position statement of The North American Menopause Society. Menopause,10:113-32.

Van Baal W M, Kenemans P, van der Mooren MJ, et al. 1999. Increased Creactive protein levels during short-term hormone replacement therapy in healthy postmenopausal women. Thromb Haemost, 81:925-8.

Vehkavaara S, Hakala-Ala-Pietilä T, Virkamäki A, et al. 2000. Differential effects of oral and transdermal estrogen replacement therapy on endothelial function in post-menopausal women. Circulation, 102:2687-93.

Vongpatanasin W, Tuncel M, Wang Z, et al. 2003. Differential effects of oral versus transdermal estrogen replacement therapy on C-reactive protein in postmenopausal women. J Am Coll Cardiol, 41:1358-63.

Wakatsuki A, Okatani Y, Ikenoue N, et al. 2002. Different effects of oral conjugated equine estrogen and transdermal estrogen replacement therapy on size and oxidative susceptibility of low-density lipoprotein particles in post-menopausal women. Circulation, 106:1771-6.

Weissberger AJ, HO KKY, Lazarus L. 1991. Contrasting effects of oral and transdermal routes of estrogen replacement therapy on 24-hour growth hormone $(\mathrm{GH})$ secretion, insulin-like growth factor I, and GH-binding protein in post-menopausal women. J Clin Endocrinol Metab, 72:374-81. 